\title{
Valosin-containing protein regulates the proteasome-mediated degradation of DNA-PKcs in glioma cells
}

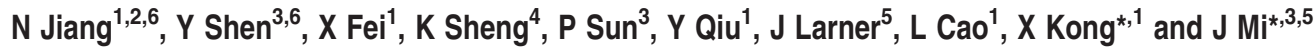

DNA-dependent protein kinase (DNA-PK) has an important role in the repair of DNA damage and regulates the radiation sensitivity of glioblastoma cells. The VCP (valosine-containing protein), a chaperone protein that regulates ubiquitin-dependent protein degradation, is phosphorylated by DNA-PK and recruited to DNA double-strand break sites to regulate DNA damage repair. However, it is not clear whether VCP is involved in DNA-PKcs (DNA-PK catalytic subunit) degradation or whether it regulates the radiosensitivity of glioblastoma. Our data demonstrated that DNA-PKcs was ubiquitinated and bound to VCP. VCP knockdown resulted in the accumulation of the DNA-PKcs protein in glioblastoma cells, and the proteasome inhibitor MG132 synergised this increase. As expected, this increase promoted the efficiency of DNA repair in several glioblastoma cell lines; in turn, this enhanced activity decreased the radiation sensitivity and prolonged the survival fraction of glioblastoma cells in vitro. Moreover, the VCP knockdown in glioblastoma cells reduced the survival time of the xenografted mice with radiation treatment relative to the control xenografted glioblastoma mice. In addition, the VCP protein was also downregulated in 25\% of GBM tissues from patients (WHO, grade IV astrocytoma), and the VCP protein level was correlated with patient survival $\left(R^{2}=0.5222, P<0.05\right)$. These findings demonstrated that VCP regulates DNA-PKcs degradation and increases the sensitivity of GBM cells to radiation.

Cell Death and Disease (2013) 4, e647; doi:10.1038/cddis.2013.171; published online 30 May 2013

Subject Category: Cancer

Valosin-containing protein (VCP), is a member of the AAA class of ATPases and associated with diverse cellular activities. ${ }^{1,2}$ VCP is an abundant protein in the cytosol and nucleus, ${ }^{3,4}$ and physiologically exists as a homohexamer. Most, if not all, hexamers make further complexes with other adaptor proteins, such as p47 or Ufd1-Npl4, , 5 and escort ubiquitin-protein conjugates to the proteasome through protein-protein interactions in order to prevent the formation of excessive multiubiquitin chain sizes. ${ }^{2}$ Moreover, recent data show that VCP also regulates protein ubiquitination by activating a deubiquitinating enzyme, Ataxin- $3{ }^{7}$ It is believed that VCP is directed to relevant cellular processes through differential binding to specific adaptor proteins. ${ }^{8}$ Each VCP protomer is composed of four regions: the N-terminal, two ATPase (D1 and D2) and the C-terminal regions. ${ }^{1,9}$ The $\mathrm{N}$-terminal region possesses binding activities to the adaptor proteins as well as ubiquitinated proteins. ${ }^{1}$ VCP has been proposed to perform many physiological cellular functions, such as the protein degradation mediated by the ubiquitin-proteasome system. ${ }^{5,10-14}$

Interestingly, VCP has also been reported to be recruited to DNA double-strand break (DSBs) sites by interacting with
BRCA1, p53 and RAD51, and it might regulate DNA damage repair. ${ }^{4,15,16}$ A recent study has revealed that VCP is a novel substrate of DNA-dependent protein kinase (DNA-PK) and other PIKK family members. ${ }^{17}$ In response to DNA damage, VCP is phosphorylated at Ser 784 within its $\mathrm{COOH}$ terminus, a region shown to target VCP to specific intracellular compartments; VCP is then accumulated at the sites of DSBs with the ubiquitin ligase RNF8, ubiquitin adaptor UFD1-NPL4 and Lys-48-linked ubiquitin (K48-Ub) chains. ${ }^{16}$ The DNA damage-induced phosphorylation of VCP and its recruitment to DSBs sites suggest a role for VCP in DNA repair.

The DNA-PK is a DNA-activated serine/threonine protein kinase conserved in vertebrates. ${ }^{18}$ DNA-PK is composed of a catalytic subunit (DNA-PKcs) and one Ku heterodimer, ${ }^{19}$ and it is ubiquitously expressed in almost all mammalian cells. Numerous studies have shown that DNA-PK has essential roles in the repair of DSBs, especially in non-homologous end joining (NHEJ). Moreover, DNA-PK not only has a critical role in the DNA damage repair pathway, but it is also involved in other important physiological or pathological processes, ${ }^{20}$ such as radiation sensitivity, inflammatory responses and

\footnotetext{
${ }^{1}$ Renji Hospital, Shanghai Jiao Tong University School of Medicine, Shanghai, China; ${ }^{2}$ Institute of Cancer Stem Cells, Dalian Medical University, Dalian, China; ${ }^{3}$ Department of Biochemistry and Molecular Cell Biology, Key Laboratory of Tumor Microenvironment and Inflammation, Shanghai Jiao Tong University School of Medicine, Shanghai, China; ${ }^{4}$ Department of Radiation Oncology, UCLA, Los Angeles, CA, USA and ${ }^{5}$ Department of Radiation Oncology, University of Virginia, Charlottesville, VA, USA *Corresponding author: X Kong, Renji Hospital, Shanghai Jiao Tong University School of Medicine, 1630 Dongfang Rd., Shanghai 200127, China. Tel: + 8621 58752345; Fax: + 8621 63260930; E-mail: kxm666@sohu.com.

or J Mi, Department of Biochemistry and Molecular Cell Biology, Key Laboratory of Tumor Microenvironment and Inflammation, Shanghai Jiao Tong University School of Medicine, 280 South Chongqing Road, Shanghai 200025, China. Tel: 86-21-63846590 + 776491; Fax: 86-21-54660873; E-mail: jmei@sjtu.edu.cn

${ }^{6}$ These authors contributed equally to this work.

Keywords: VCP; GBM; radiation resistance; DNA-PK

Abbreviations: VCP, valosin-containing protein; DNA-PK, DNA-dependent protein kinase; ER, endoplasmic reticulum; ERAD, ER-associated protein degradation; DSB, DNA double-strand break; VIM, VCP-interacting motif; NHEJ, non-homologous end joining; ATR, ATM-Rad3-related protein; ATM, ataxia telangiectasia mutated protein; CPT, camptothecin

Received 26.1.13; revised 12.4.13; accepted 15.4.13; Edited by A Stephanou
} 
metabolic gene expression. ${ }^{21-23}$ However, it is still unknown how DNA-PK protein degradation is regulated and whether VCP affects the radiation sensitivity.

In this study, we investigated the association of VCP with DNA-PKcs and the effect of the regulation of VCP on the radiosensitivity of glioblastoma cells. Our data demonstrated that DNA-PKcs was ubiquitinated and bound to VCP; the VCP knockdown resulted in the accumulation of DNA-PKcs protein in glioblastoma cells and increased DNA-PK activity, which eventually increased the survival fraction of GBM cells post-IR and shortened the survival time of orthotopic xenografted mice.

\section{Results}

VCP is associated with DNA-PK and the D1 domain of VCP is essential for this binding. According to bioinformatic analysis, the DNA-PKcs protein contains a VCP-interacting motif (VIM), which is conserved in mammals such as humans, monkeys, cows and wolves (Figure 1a). To determine whether VCP is associated with DNA-PK, co-immunoprecipitation (co-IP) was performed using the DNA-PKcs antibody in several glioblastoma cell lines; the M059J cell is DNA-PKcs-deficient, and was used as a negative control. Western blots following co-IP showed that VCP is pulled down with DNA-PKcs (Figure 1b); moreover, in agreement with our previous study, ${ }^{24}$ the more sensitive the cell is, the more VCP associates with DNA-PK, indicating that VCP may be related to radiation resistance. However, radiation itself does not significantly affect the binding of VCP with DNA-PKcs (data not show).
To further determine which domain of VCP is essential for this binding, the plasmids containing different VCP domains were separately transfected into 293T cells. Including an empty vector, seven plasmids, as outlined in the schematic drawing in Figure 1c, were generously provided by Dr. Ueffing. ${ }^{25}$ Two days after transfection, whole lysates from 293T cells were subjected to western blot analysis, and $1 \mathrm{mg}$ of total protein was used for IP against the flag antibody. Western blots following IP showed that the full-length, the isolated $\mathrm{N}$ domain and the isolated D1 domain, but not the D2 domain, can directly bind to DNA-PK, and only the ND1 domain of the fusion proteins can bind with DNA-PK. The ND2 fusion proteins have no detectable binding with DNA-PK, and the D1D2 fusion protein also weakens the binding affinity of the D1 domain to DNA-PK. These observations suggest that the D2 domain has a negative influence on the binding of DNA-PK with VCP, which may be mediated by multiple mechanisms, including mutually exclusive binding or conformational changes.

In addition, the full-length VCP-immunoprecipitated DNA-PKcs protein was less than that immunoprecipitated by the isolated $\mathrm{N}$ domain or D1 domain; moreover, the total DNAPKcs protein in the full-length VCP-transfected 293T cells also decreased. These observations suggest that the reduced signal most likely resulted from the decreased total DNA-PKcs protein, and that the overexpression of VCP might have caused the degradation of DNA-PKcs. The western blot for whole-cell lysates showed that all truncates containing VCP domains were equally overexpressed (bottom panel of Figure 1d).

\section{a}

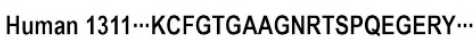

Cow $1311 \cdots$ KCFGTGAAGNRTSPQEGERY.*

Wolf $1323 \cdots$ KCFGTGAAGHRPSPQEERY...

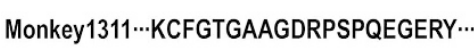

VIM

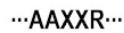

C

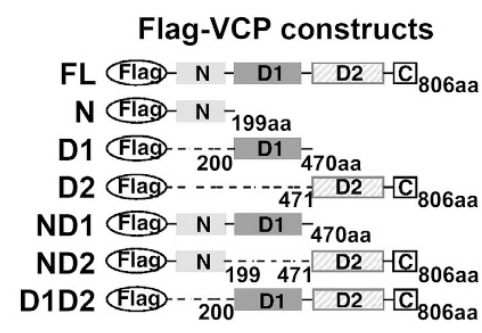

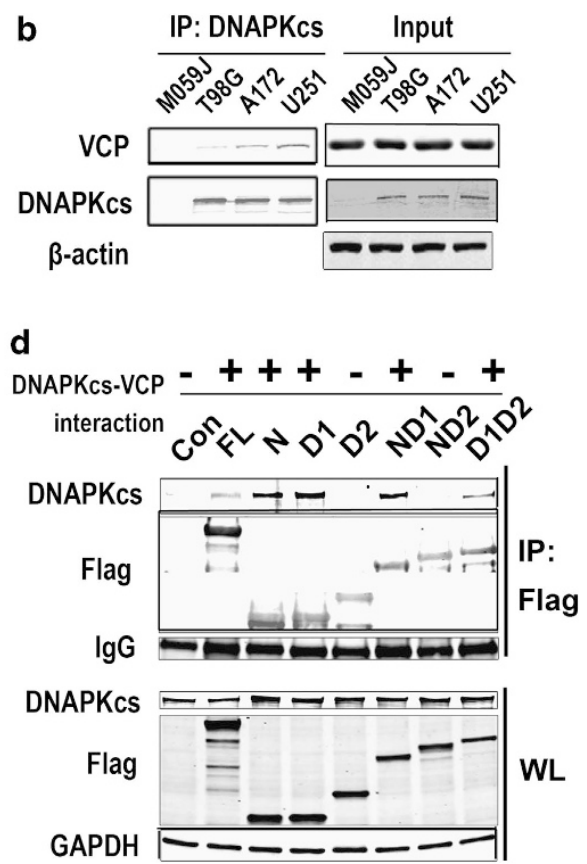

Figure 1 VCP was associated with DNA-PK. (a) The VIM and the protein sequence alignment among humans, monkeys, cows and wolves. (b) The VCP was pulled down by DNA-PKcs IP in different GBM cell lines (WL: whole-cell lysates). DNA-PKcs-deficient M059J cells were considered as negative controls. (c) The schematic drawing of the truncated VCP protein. All truncates were tagged with the Flag epitope (FL: full-length). (d) The plasmids expressing different domains of VCP were separately transfected into 293T cells (con: empty vector), and flag immunmoprecipitation was performed. Both DNA-PKcs and flag were detected in whole-cell lysates (bottom panels, shown as WL) or flag IPs (top panels) 
DNA-PKcs degradation is ubiquitin-proteasome-dependent and regulated by VCP. To determine whether DNA-PK protein degradation is dependent on proteasomes and whether VCP regulates DNA-PK degradation, VCP was knocked down by lentivirus-mediated shRNA (this shRNA was verified by the other group ${ }^{26}$ ) in U251 cells or U87 cells. Western blots showed that DNA-PKcs was upregulated in VCP-knockdown cells, while the overexpression of VCP reduced the amount of DNA-PKcs (Figure 2a), which indicates that VCP regulates the DNA-PKcs protein level. Regarding of the function of VCP, VCP might affect DNAPKcs degradation. To exclude the possibility that VCP regulates the transcription of DNA-PKcs, quantitative PCR (q-PCR) was performed in order to assess the level of DNA-PKcs mRNA. The level of DNA-Pkcs mRNA was detected in VCP-knockdown cells, control cells and parental cells (Figure 2b). As expected, there was no significant difference in DNA-PKcs mRNA levels among VCP knockdown U87, control U87 or parental U87 cells, which suggests that VCP might regulate DNA-PKcs degradation instead of modulating its transcription.

Because the VCP-mediated protein degradation is ubiquitin-proteasome-dependent, to determine whether
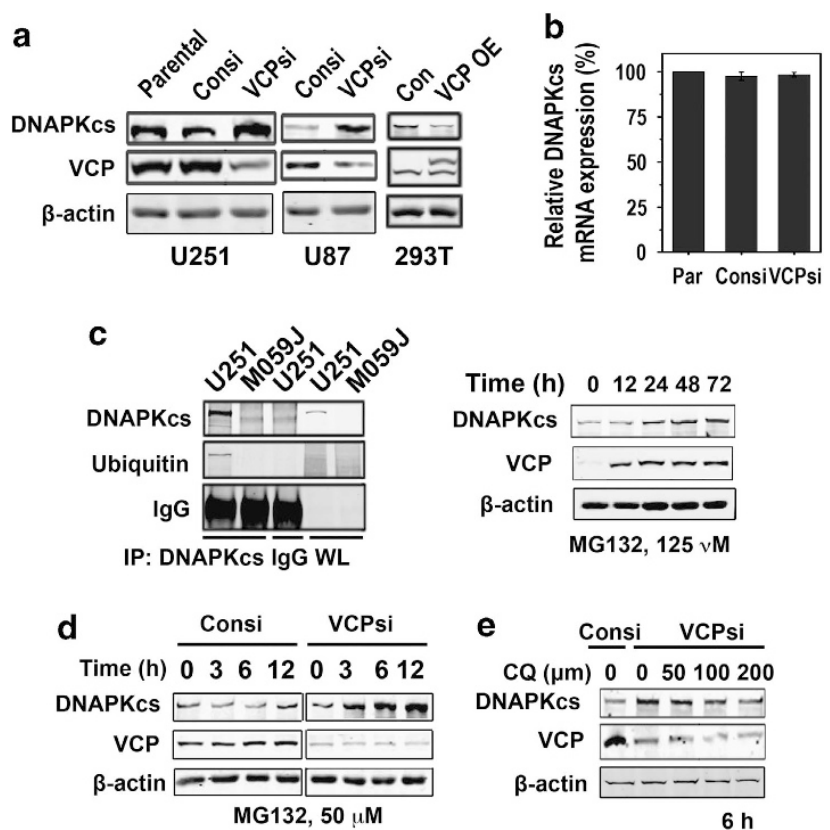

Figure 2 VCP regulated DNA-PKcs degradation and this degradation was ubiquitin-proteasome-dependent. (a) U251 or U87 cells were infected with VCP shRNA lentiviruses or VCP expression lentiviruses. Immunoblots were performed against whole-cell lysates (Par: parent U251 cells; Consi: nonspecific RNA; VCPsi: VCP-specific siRNA). (b) The real-time PCR was performed using DNA-PKcsspecific primer in U251 cells infected with different lentiviruses (Par: parent U251 cells); DNA-PKcs mRNA levels are relative to GAPDH. (c) Top panel: DNA-PKcs was immunoprecipitated, and both DNA-PKcs and ubiquitin were detected on the same membrane (WL: whole-cell lysates). Bottom panel: DNA-PKcs was detected in parent U251 cells treated with MG132 (125 nM) for the indicated dose. (d) Parent U251 cells or U251 cells infected with different lentiviruses were treated with the proteasome inhibitor MG132 $(50 \mu \mathrm{M})$, and the DNA-PKcs protein was detected at the indicated time points. (e) Parent U251 cells or cells infected with different lentiviruses were treated with the lysosome inhibitor chloroform at the indicated concentrations, and the DNA-PKcs protein was detected $12 \mathrm{~h}$ after treatment
DNA-PKcs is ubiquitinated, DNA-PKcs was purified via IP and followed by ubiquitin detection. The western blot showed that the ubiquitin signal only appeared in the DNA-PKcsproficient U251 cells at the size of DNA-PKcs, but not in the DNA-PKcs-deficient M059J cells or nonspecific IgG lane, which indicates that the DNA-PKcs was ubiquitinated (Figure 2c). To further identify whether DNA-PKcs degradation is dependent of the proteasome system, U251 cells were treated with the proteasome inhibitor MG132 (125 nM) and harvested at the indicated time points $(0,12,24,48,72 \mathrm{~h})$ based on the half-life of the DNA-PKcs protein.

The western blot data showed that the DNA-PKcs protein level was significantly increased $24 \mathrm{~h}$ after MG132 treatment; the longer the treatment lasted, the more the DNA-PKcs accumulated (Figure 2c), which indicates that the DNA-PKcs degradation was dependent on the ubiquitin-proteasome system, although the typical smeared bands were not seen, perhaps due to the high-molecular weight of DNA-PKcs (460 KD). To determine whether VCP knockdown has synergistic effects on DNA-PKcs accumulation with proteasome inhibition, the control and the VCP-knockdown U251 cells were treated with MG132 for the indicated time $(0,3,6$, $12 \mathrm{~h})$. The immunoblots showed that the DNA-PKcs was dramatically increased in VCP-knockdown U251 cells $3 \mathrm{~h}$ after MG132 treatment (Figure 2d). Moreover, to exclude the possibility that this increase was dependent on lysosomes, VCP-knocked down U251 cells were treated with the lysosome inhibitor chloroquine at the indicated concentration. The western blot showed that chloroquine had no effects on DNA-PKcs accumulation, even $6 \mathrm{~h}$ after treatment; in contrast, chloroquine decreased the protein levels of both DNA-PKcs and VCP (Figure 2e). In brief, our data demonstrated that DNA-PKcs was ubiquitinated and that VCP regulated DNA-PKcs degradation through the ubiquitin-proteasome system.

Knockdown of VCP enhances the DNA-PK activity and increases the efficiency of DNA damage repair. To determine whether $\mathrm{VCP}$ regulates DNA-PK activity as well as DNA-PKcs protein levels, the endogenous DNA-PK activity was directly assessed via in vitro kinase assay, and indirectly measured through the phosphorylation of endogenous RPA32 (the 32-kDa subunit of replication protein A). Although RPA32 is differentially phosphorylated by three PI3Ks (ataxia telangiectasia mutated protein, ATM, ATM-Rad3-related protein and DNA-PK) in response to different DNA damaging agents, DNA-PK is the primary kinase responsible for camptothecin (CPT)-induced RPA32 phosphorylation; ${ }^{27-29,24}$ thus, CPT-induced RPA32 phosphorylation is another indicator of DNA-PK activity.

In the in vitro kinase assay, our data showed that DNA-PK was activated by ionising radiation and that VCP knockdown enhanced this activation, which was one and a half times more than that in control cells, as shown in the first graphic of Figure 3a. In response to CPT treatment, the Ser 4/8 phosphorylation of RPA32 was observed as the appearance of a band of reduced mobility relative to the parent RPA32 band, and this shifted band was confirmed by the phosphorylation-specific antibody. This CPT-induced RPA32 phosphorylation in parental U251 cells, U251 cells with VCP 

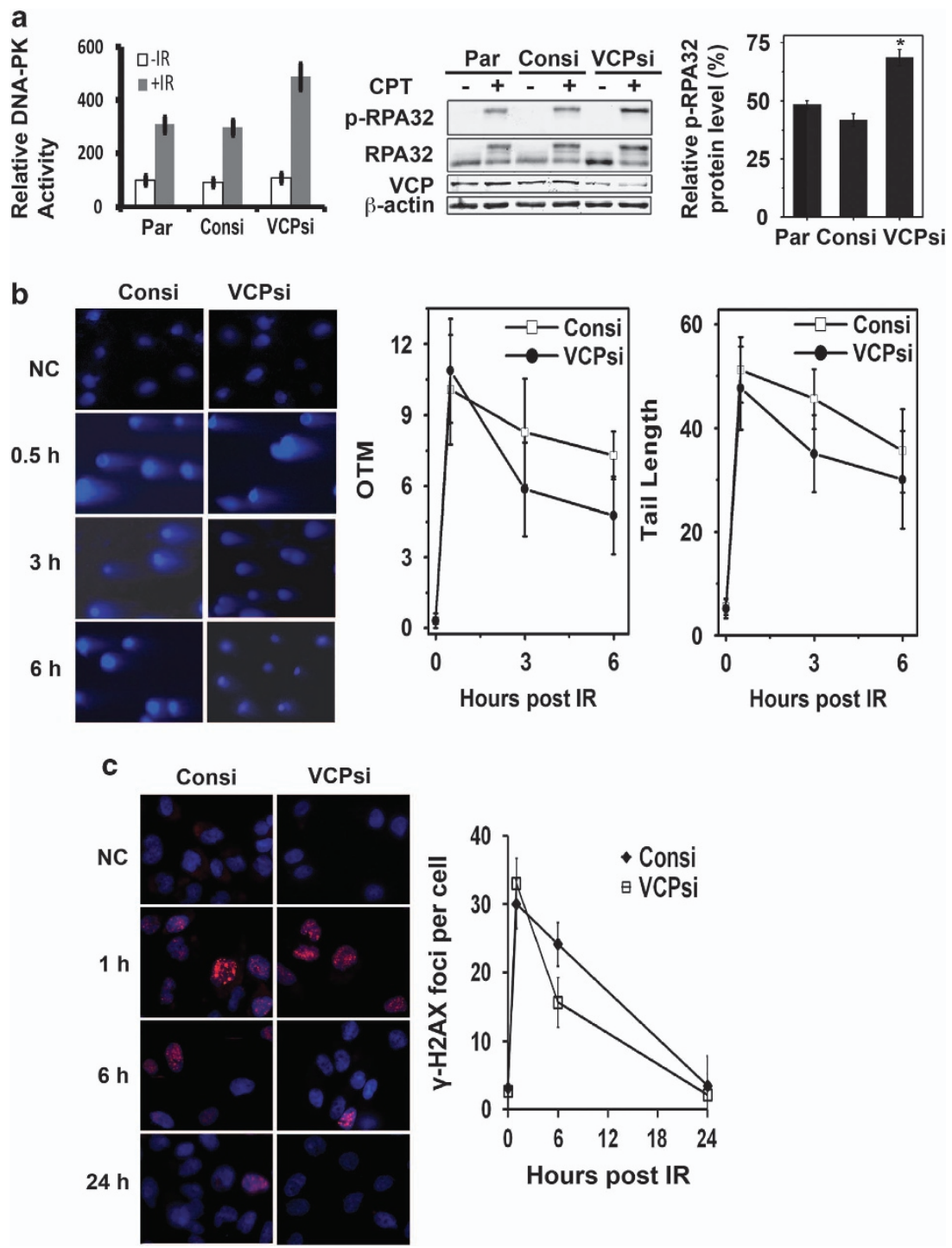

Figure 3 VCP knockdown increased DNA-PK activity and promoted the efficiency of DNA damage repair. (a) Parent U87 cells or cells infected with control lentiviruses or VCP shRNA lentiviruses were treated with 5 Gy of radiation or camptothecin $(20 \mu \mathrm{M})$. The in vitro kinase assay was performed on cells treated with radiation. Cells treated with CPT were subjected to immunoblot, and ser $4 / 8$ phosphorylation of RPA32 was detected by densitometry analysis $2 \mathrm{~h}$ after the treatment (the columns present the mean \pm S.D., three independent experiments were performed). The ratio of phosphor-RPA32 to total RPA32 was calculated, and the value was normalised to the $\beta$-actin density. (b) U87 cells infected with control lentiviruses or VCP shRNA lentiviruses were treated with 5 Gy of radiation; the cells were harvested at indicated time points for comet assay, and 100 cells in each group were measured for the value of OTM and tail length. (c) U87 cells with control lentiviruses or VCP shRNA lentiviruses were immunostained with $\gamma-\mathrm{H} 2 \mathrm{AX}$ after receiving $2 \mathrm{~Gy}$ radiation; 100 cells were counted, and the graphic presents the average number of foci per cell at the indicated time points (red: $\gamma$-H2AX; blue: DAPI). (Par: parent U87 cells; Consi: nonspecific RNA; VCPsi: VCP-specific siRNA)

knockdown or control siRNA (Figure 3a) were analysed by densitometry using ImageJ software (NIH, USA). The ratio of phosphorylated RPA32 (top panel) to total RPA32 (two bands in the second panel), which represents DNA-PK activity, was calculated in each lane. The ratio of p-RPA32 in the VCP-knockdown U251 cells was two times more than that in the control U251, as shown in the last graphic of Figure 3a. The total RPA32 was increased in the VCP-knockdown cells, but the cause of this is unknown.

Moreover, the COMET assay and $\gamma-\mathrm{H} 2 \mathrm{AX}$ detection, which represent the DNA damage level in each cell, were performed in order to assess the efficiency of DNA damage repair. In the
COMET assay, the cells were irradiated with 10 Gy radiation on ice. Treated cells were harvested and recovered for the indicated time $(30,180,360 \mathrm{~min})$, and the cells were subjected to single-cell electrophoresis under neutral conditions. Each time, 200 cells for each group were measured at 200 times magnification. The tail lengths were measured and Olive tail moment (OTM) values were calculated using CASP software based on the formula: OTM $=$ tail DNA $\% \times$ (tail mean - head mean). After $30 \mathrm{~min}$, there was no significant difference between the control U251 cells and the VCP-knockdown cells. However, after 3 or $6 \mathrm{~h}$ of recovery, the tail lengths or the OTM values were obviously longer or greater in control U251 
cells compared with that in the VCP-knockdown U251 cells $(P<0.05)$, as shown in Figure $3 \mathrm{~b}$, which suggests that VCP knockdown promoted DNA repair efficiency.

Furthermore, another DNA damage marker, $\gamma-\mathrm{H} 2 \mathrm{AX}$ foci, was also counted in the U251 cells treated with 2 Gy of radiation (the data with other doses of radiation are not shown). One hour after radiation, massive $\gamma$-H2AX foci were observed in both the U251 control cells and VCP-knockdown cells, and almost every single cell contained numerous foci (red dots). Six hours after radiation, most control cells had no dramatic changes in $\gamma-\mathrm{H} 2 \mathrm{AX}$ staining, while the foci in half of the VCP-knockdown cells had disappeared $(P<0.01)$ (Figure 3c). Eventually, both control and VCP-knockdown cells had no foci detectable after $24 \mathrm{~h}$ of recovery. All these observations demonstrate that VCP knockdown enhances the DNA-PK activity and increases the efficiency of DNA damage repair.

VCP knockdown increases the survival fraction of GBM cells after radiation treatment and shortens the survival of orthotopic xenografted mice. To determine whether VCP regulates the radiation sensitivity of GBM cells, an in vitro clonogenic assay and in vivo orthotopic mouse model were used in this study. In the clonogenic assay, signalised VCP-knockdown U87 cells or U251 cells were seeded in $100-\mathrm{mm}$ dishes (400 cells each); the cells were irradiated at the indicated dose and then were cultured for 2 weeks. The colonies containing 50 cells or more were counted. VCP knockdown increased the survival fraction in both U251 cells and U87 cells. After 2 Gy of radiation, VCP knockdown promoted the survival of U251 cells from 29.3 to $51.3 \%$ $(P<0.01)$ and the survival of U87 cells from 34.9 to $62.3 \%$ $(P<0.01)$ (Figure $4 \mathrm{a})$. U87 cells were more resistant to radiation than U251 cells, which was consistent with our previous study. ${ }^{24}$ There were no significant effects on survival between the control and parental U251 or U87 cells. To further confirm whether VCP knockdown increased radiation resistance is dependent on DNA-PK, a pair of DNA-PKcs-proficient (M059K) and deficient-(M059J) GBM cells were used in this study. Our data showed that VCP knockdown significantly enhanced the survival of DNA-PK-proficient M059K cells, but not DNA-PK-deficient M059J cells, demonstrating that VCP knockdown-produced radiation resistance is DNA-PK-dependent.

In the orthotopic mouse model, U87 cells infected with empty viruses served as controls, and 48 mice in total were injected. Nine days after the injection, mice bearing tumours (39 mice) were randomly divided into two groups according to the MRI imaging, including IR and non-IR irradiation. Each group contained control and knockdown subgroups (10 mice in each subgroup). The $6 \mathrm{~Gy}$ of total radiation were administered three times over 1 week; the schedule is described in the Materials and Methods section. The survival time of each mouse was recorded, and all survival times in each group were analysed using the Kaplan-Meier method. Our data showed that the radiation treatment prolonged the mice's survival time in all groups. With radiation treatment, the mice in the VCP-knockdown group survived for a shorter time (38.4 days, median survival time) than the mice in control groups, whose survival time was 53.8 days (Figure $4 \mathrm{~b}$ ).
Without radiation treatment, the survival time in the VCPknockdown group was slightly shorter than that in the control group. The radiation enhancement ratio of survival time in the VCP-knockdown group was $2.69 \%$, much lower than that in the control group (27.1\%); the difference was statistically significant $(P<0.001)$ (Figure 4b).

On the basis of the MRI image of tumours at the 9th day after intracranial injection, the tumour size in each group differed, but not significantly, as seen in the representative pictures in the middle graphic of Figure 4c. As we expected, the tumour sizes in the VCP-knockdown group at the 29th day were obviously larger than that in the same mouse at the 9th day, as shown in the representative images in the right column of middle graphic (Figure 4c). The average tumour volumes in each group were calculated and are shown in Figure $4 \mathrm{c}$. The tumour sizes shrunk more $(28.9 \%)$ in the control group than in the VCP-knockdown group (6.3\%) after radiation treatment. The representative fluorescence immunostaining pictures of VCP protein levels in the xenografted GBM are shown in Figure 4c.

To further investigate VCP expression and its effects on the radiation sensitivity of GBM, the VCP protein expression in GBM sections from patients (grade IV astrocytoma) were semiquantified with Tissue IA quantitative analysis software (Leica, Solms, Germany), and the correlation with survival time was analysed. All digital images were normalised to the negative control. Scores less than $10 \%$ were considered to be '- '; the scores greater than $10 \%$ and less than $25 \%$ were considered to be ' + '; and scores more than $25 \%$ were considered to be ' ++ '. The data showed that VCP was differentially expressed in the GBM tissue and that the VCP expression level was positively correlated with patients' survival time $\left(R^{2}=0.5222, P<0.05\right)$. Patients bearing high levels of VCP would survive longer with radiation treatment (Figure 4d).

The representative pictures are shown in Figure $4 \mathrm{e}$. This observation was consistent with our animal data.

\section{Discussion}

We first found that the DNA-PKcs is ubiquitinated and bound to VCP; the treatment with proteasome inhibitor (MG132), but not lysosome inhibitor (chloroquine), increased the protein level of DNA-PKcs; the VCP knockdown had a synergistic effect with the MG132 treatment on DNA-PKcs protein accumulation. As a carrier, VCP binds to polyubiquitinated protein and delivers it to the proteasome system for degradation; thus, either proteasome inhibition or VCP knockdown increased the DNA-PKcs protein level, and the combination of both synergistically increased the DNA-PKcs protein level. These observations demonstrated that VCP regulates DNA-Pkcs protein degradation, which is ubiquitinproteasome-dependent. Interestingly, MG132 treatment also increased the level of the VCP protein itself, which suggests that the degradation of VCP is also regulated by the ubiquitinproteasome system. Moreover, chloroquine treatment unexpectedly decreased the protein level of both DNA-PKcs and VCP in glioblastoma cells, which indicates that lysosome inhibition may enhance proteasome degradation or endoplasmic reticulum-associated degradation in a compensatory way. 

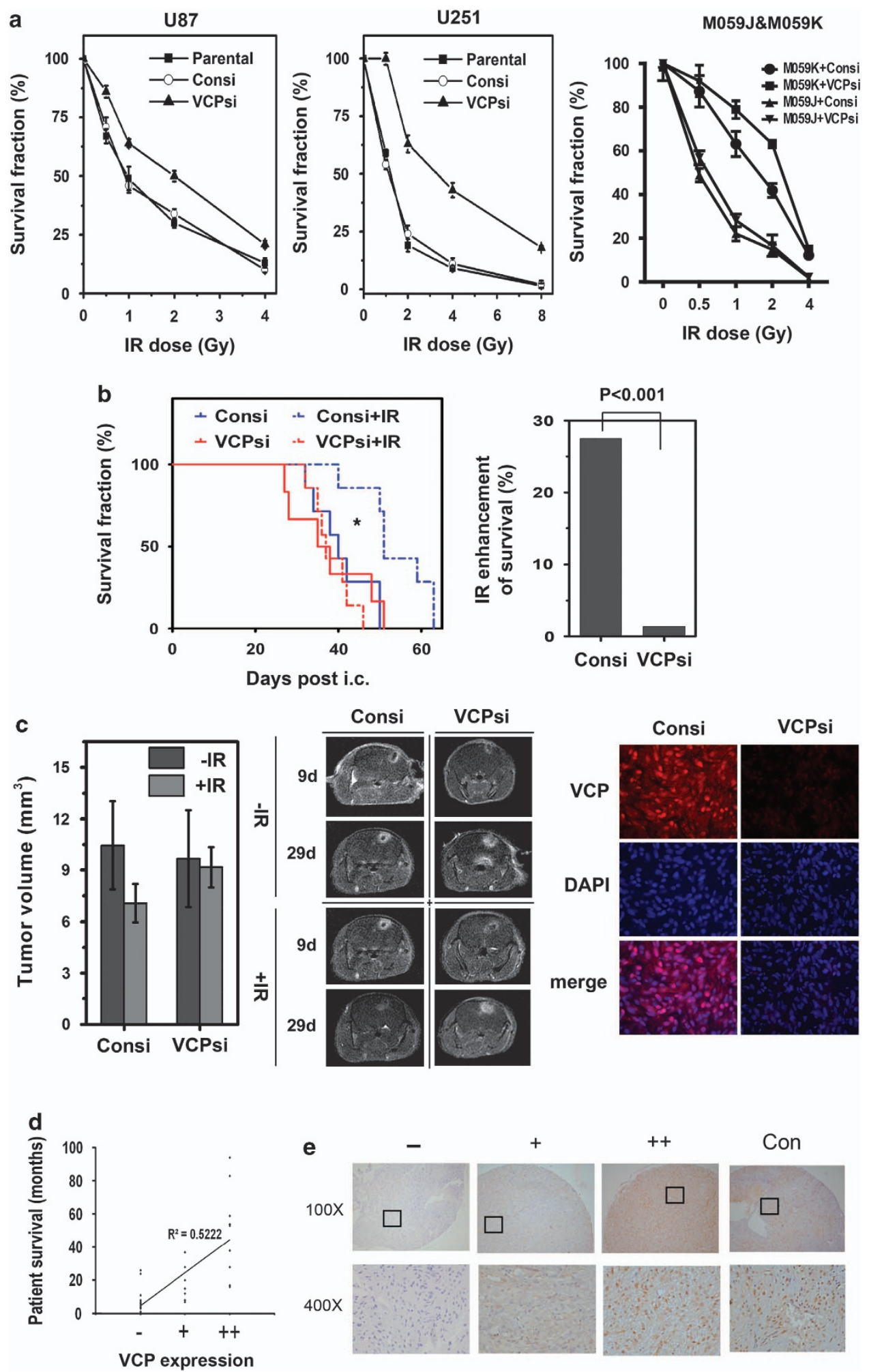

Con

Figure 4 VCP knockdown increased the radiation resistance of GBM cells. (a) Exponentially growing parent U251, U87, M059K, M059J cells or cells infected with control lentiviruses or VCP shRNA lentiviruses were separately seeded in 10-cm dishes (400 cells per dish), clonogenic assays were performed following radiation treatment at the indicated doses, and the survival fraction were normalised to control cells. (b) the Kaplan-Meier survival curve was made for orthotopic GBM mice in four groups $\left({ }^{*} P<0.05\right)$; the radiation enhancement of survival time was calculated based on the formula: the radiation enhancement of survival time $=$ the extended survival time/the survival time without radiation treatment. (c) The average tumour size and the representative MR images in each group and representative VCP immunofluorescence images (red) in xenografted glioblastoma sections. (d) Correlation analysis of VCP protein levels with patient survival, and the representative VCP immunohistochemistry images in paraffin sections from glioblastoma patients. (e) Representative pictures from each group (Con: para-tumour tissue) 
Furthermore, although DNA-PK is thought to be a nucleolus protein, our observation suggests that DNA-PK, or at least its catalytic subunit, is also located in the cytoplasm, ${ }^{21}$ and that VCP associated with it and regulated its degradation. The coIP experiment identified that both the $\mathrm{N}$-terminal domain and the D1 domain of the VCP protein could bind to DNA-PK, which is consistent with the recent findings of co-IP and biolayer interferometry experiments using a VIM peptide of gp78; ${ }^{30}$ this conclusion was also partially consistent with early data that showed that the rhodopsin $(\mathrm{P} 23 \mathrm{H})$ protein only interacts with the p97ND1 domain, but not the isolated p97N domain. ${ }^{25}$ However, the VCP ND2 domain exhibits no detectable binding with DNA-PKcs, and the D1D2 domain also weakens the binding affinity of the D1 domain with DNAPKcs. These results indicate that VCP association with the target protein is affected by conformational changes, which may be induced by ATP binding/hydrolysis or hierarchical binding. Moreover, it was reported that one single amino-acid substitution $(\mathrm{R} 155 \mathrm{H})$ at the interface between the $\mathrm{N}$-terminal domain and the adjacent AAA domain (D1) of VCP results in a reduced affinity for ADP. ${ }^{31}$ Although the previous data showed that VCP was phosphorylated by DNA-PK in response to DNA damage, it is not clear whether this phosphorylation of VCP affects its binding with DNA-PK or regulates VCP activation.

In addition, biochemical and structural studies have demonstrated that multiple cofactors recognised by VCP (p97) contain the ubiquitin regulatory $X$ (UBX) domain present in the UBX protein family or the ubiquitin-like domain of NPL4. ${ }^{30}$ In addition to the fact that VCP is phosphorylated at Ser 784, DNA-PKcs also contains the VIM motif of 'AAXXR' (Figure 1a), which is conserved in mammals and is consistent with the previous finding. ${ }^{30}$ Our data also showed that DNA-PKcs is ubiquitinated and its degradation is ubiquitinproteasome-dependent; however, the specific ubiquitylated site(s) of DNA-PKcs and its corresponding E3 ligase have not been discovered yet and will be addressed in our future studies.

DSB is a most lethal type of DNA damage; if the cell is unable to fix this damage, the unrepaired DSBs may result in cell death. As a central component of the NHEJ, DNA-PK controls the process of DSB repair and maintains the stability of the genome. Alterations in DNA-PK activities or amounts of the DNA-PKcs protein will most likely change the sensitivity of cells to genotoxic stress, such as radiation. It is well known that DNA-PKCs ${ }^{-/-}$mice are hypersensitive to radiation and undergo ATM- and p53-dependent apoptosis. ${ }^{32-34}$ However, data from TCGA and our group suggest that there is no significant difference in the DNA-PKcs protein levels between normal glial tissue and GBM tumour tissue, although in some cases, immunohistochemistry analysis showed that tumour cells express more DNA-PKcs than adjacent normal tissue, ${ }^{35}$ which may be due to the tissue specificity.

To avoid potential side effects caused by DNA-PK inhibitors, targeting DNA-PK regulatory proteins that are upregulated in radiation-resistant glioblastoma cells is an alternative strategy for radiosensitisation treatment. We previously found that the protein phosphatase PP6 regulates the radiation sensitivity of GBM cells through DNA-PK, and that knocking down PP6c increases the survival time of mice; moreover, PP6c was overexpressed in $44.7 \%$ (17 of 38 cases) of GBM patients. ${ }^{21,22,36,37,24}$

In this study, knocking down VCP not only caused the accumulation of the DNA-PKcs protein, but it also elevated the DNA-PK activity and promoted the efficiency of DNA damage repair in GBM cells. Although VCP knockdown affected 53BP1 recruitment into the DNA damage sites and seemed to increase the radiation sensitivity of U2OS cells and nematode, ${ }^{15,16}$ our data showed that the accumulation of DNA-PK increased the efficiency of DNA damage repair in GBM cells, which might be a result of p53-independent pathways and a different cellular roles of VCP in different types of cells. ${ }^{38}$ Our in vivo study confirmed that VCP knockdown reduced the radiosensitivity and shortened the survival time of mice in a GBM orthotopic model. The clinical data also supported this conclusion. Therefore, the small molecules that selectively target the VCP protein could influence the radiation sensitivity by regulating DNA-PK protein level. These observations suggest that DNA-PK regulatory proteins are potential targets for radiosensitisation treatment.

\section{Materials and Methods}

Human subjects and tumour samples. The research protocol was approved by the Institutional Review Board of the Shanghai Jiao Tong University School of Medicine. A total of 38 GBM patients were recruited in the affiliated Renji Hospital neurosurgical clinic and provided informed consent; patients with newly diagnosed, histologically confirmed GBM (World Health Organisation grade IV astrocytoma) were eligible for this study. These eligible patients received standard radiotherapy (fractionated focal irradiation in daily fractions of 2 Gy given 5 days per week for 6 weeks) without chemotherapy within 1 month after the surgery for subtotal removal. The primary end point was patient's death.

The orthotopic GBM mouse model. Congenitally athymic nude mice, five to six weeks old (Charles River Laboratories, Wilmington, MA, USA), were used in this study, which was approved by the Shanghai Jiao Tong University Animal Care and Use Committee. Under deep isoflurane anaesthesia, mice were placed in a small-animal stereotactic frame (David Kopf Instrument, Tujunga, CA, USA). A sagittal incision was made to expose the cranium, and a bur hole was made in the skull, $0.2 \mathrm{~mm}$ anterior and $1.8 \mathrm{~mm}$ lateral (right), from the bregma using a small dental drill. At a depth of $3 \mathrm{~mm}$ from the brain surface, a $1 \times 10^{6}$ cells $/ 5 \mu \mathrm{l}$ cell suspension was injected. Five minutes later, the needle was removed and the hole was sealed with bone wax. The wound was sutured immediately. The survival times of the mice were recorded. The intragroup variation in length of survival, including the S.D. and $95 \%$ confidence interval determinations, were analysed.

In vivo MRI imaging of tumour. GBM-bearing mice, with or without radiation, were examined on day 9,29 days post-tumour implantation to detect the growth of the grafted tumour fragments. Tumour imaging was performed with a small-animal coil on a high-field GE Signa 3-tesla clinical MR scanner (General Electric, Waukesha, WI, USA), and images were obtained using a standard T1 protocol following an intraperitoneal injection of gadolinium (Gd-DTPA, $100 \mu \mathrm{l} / 20$ g, Bayer Inc., Pittsburgh, PA, USA) 10 min before examination. In enhanced scanning, the scanning parameters were: AxT1 FSE series: Scan plane: oblique; FOV: 5.0; phase FOV: 0.60; slice thickness: $1.0 \mathrm{~mm}$; spacing: $0.0 \mathrm{~mm}$; Freq DIR: R/L; Auto TR: 600 , minimum TR: 60 . The tumour sizes were measured and their volumes were calculated with Function Analysis software according to the following formula: (length $x$ width $\left.{ }^{2}\right) / 2$. These results were subsequently analysed using Image Pro Plus software (Media Cybernetics, Rockville, MD, USA). ${ }^{39}$

siRNA knockdown of VCP. Exponentially growing U87 or U251 cells were infected with lentiviruses containing specific shRNA against VCP, shRNA sequence: $5^{\prime}$-CGCATTGTATCACAGTTGT- $3^{\prime}$ (sense); 5'-ACAACTGTGATACAAT GCG-3' (antisense). The preparation of lentiviruses was according to a previously described protocol. ${ }^{21}$ A nonspecific siRNA (sequence: $5^{\prime}$-AAAUCUUCGAGACAU UCUGUU- ${ }^{\prime}$ ) was used as a control. The other siRNA oligonucleotides used in this 
study were purchased from Dharmacon (Fisher scientific, Pittsburgh, PA, USA) and transfected into the cells using LipofectAMINE RNAiMAX (Invitrogen, Grand Island, NY, USA) according to the manufacturer's instructions.

Radiation treatment. Cells in culture were irradiated with an irradiator (GE 3000 ) using a ${ }^{137} \mathrm{Cs}$ source at a dose rate of $4.0 \mathrm{~Gy}$ per minute. Mice were observed daily following tumour cell injection; 10 days after xenograft transplantation, with the aid of GE HiSpeed CT, radiation was delivered accurately to the brain tumours of mice, 2 Gy each time, by an ELEKTA Precise accelerator; the body did not receive any radiation. The following radiation schedule, established in preliminary studies, was used during the course of this study: 2 Gy M-W-F for 1 week (6 Gy total administered over 7 days). All mice used for therapy response evaluations were euthanised at the time of reaching a moribund condition.

DNA-PK kinase assay. Exponentially growing cells were treated with $10 \mathrm{~Gy}$ IR. After $30 \mathrm{~min}$, the nuclear extracts were prepared as previously described. ${ }^{21}$ Briefly, the cells were permeabilised and the nuclei were extracted in lysis buffer containing $0.42 \mathrm{M} \mathrm{NaCl}$ and $1.5 \mathrm{mM} \mathrm{MgCl}$. DNA-PK activity was analysed using a DNA-PK activity assay kit ${ }^{40,41}$ according to the manufacturer's instructions (Promega Inc., Madison, WI, USA).

Immunoprecipitation. Exponentially growing U87 or U251 cells were irradiated with $10 \mathrm{~Gy} I \mathrm{R}$, then harvested at indicated time points and lysed in $1 \mathrm{ml}$ of lysis buffer (0.5\% (v/v) NP-40, $5 \mathrm{mM}$ EDTA, $2 \mathrm{mM}$ EGTA, $20 \mathrm{mM}$ MOPS, $1 \mathrm{mM}$ PMSF, $20 \mathrm{mM}$ sodium pyrophosphate, $30 \mathrm{mM}$ sodium fluoride, $40 \mathrm{mM}$ $\beta$-glycerophosphatase, $1 \mathrm{mM} \mathrm{Na}_{3} \mathrm{VO}_{3}$, and protease inhibitors) with caspase inhibitor Z-VAD-FMK. Aliquots of $1 \mathrm{mg}$ of total protein were mixed with $4 \mu \mathrm{g}$ of monoclonal anti-DNA-PKcs antibody at $4{ }^{\circ} \mathrm{C}$ overnight. The bound proteins were recovered by binding to $25 \mu$ of protein-A agarose (Sigma, St. Louis, MO, USA). The samples were separated by $7.5 \%$ SDS-PAGE gel electrophoresis and were then transferred to nitrocellulose membranes. Proteins of interest were detected with specific antibodies. Blots were scanned using an Odyssey infrared imaging system (LI-COR, Lincoln, NE, USA), and proteins were quantitatively analysed with the Odyssey software.

Comet assay. The comet assay was performed under neutral conditions according to the procedures of Singh et al. ${ }^{42}$ with modifications by Klaude et al. ${ }^{43}$ The control U251 or the U251 cells with VCP siRNA were irradiated at doses of $10 \mathrm{~Gy}$. After the radiation, the cells were allowed to repair for 30,180 and $360 \mathrm{~min}$ or were harvested immediately (time $0=$ no repair), suspended in $1.0 \%$ lowmelting agarose dissolved in PBS, and layered onto microscope slides previously covered with $0.5 \%$ normal melting point agarose. Cells were lysed at $37^{\circ} \mathrm{C}$ overnight in a buffer consisting of $2 \%$ Sarkosyl, $500 \mathrm{mM}$ EDTA, $0.5 \mathrm{mg} / \mathrm{ml}$ protein $\mathrm{K}, \mathrm{pH}$ 8.0. Next, the DNA was unwound and uncoiled in the electrophoresis buffer that consisted of $90 \mathrm{mM}$ boric acid, $90 \mathrm{mM}$ Tris $\bullet \mathrm{HCl}$ and $2 \mathrm{mM}$ EDTA(pH 8.5) for $30 \mathrm{~min}$. Then, electrophoresis was carried out in the dark, and the cells were stained with DAPI solution. The slides were examined with an Eclipse fluorescence microscope. The comet tail moment was measured in 100 cells randomly selected from each sample. Each experiment was repeated three times.

Clonogenic assay. The clonogenic assay was adapted from Franken et $\mathrm{al} .{ }^{44}$ Briefly, the IR-treated and untreated cells were harvested and 400 cells per dish were reseeded in a 100-mM dish at an appropriate density to obtain $\sim 50-100$ colonies. Following 10-14 days of incubation, cells were fixed and stained with crystal violet, and colonies containing at least 50 cells were scored.

\section{Conflict of Interest}

The authors declare no conflict of interest.

Acknowledgements. We are grateful to the reagent donators (A Griciuc and $\mathrm{M}$ Ueffing). Grant support was provided by the National Science Foundation of China (81072076), Shanghai Committee of Science and Technology (11DZ2260200), and the National Programme on Key Basic Research Project (973 Programme) (2012CB967000) to Dr. Mi and the National Science Foundation of China fund (31000349) to Dr. Shen.
1. Brunger AT, DeLaBarre B. NSF and p97NCP: similar at first, different at last. FEBS Lett 2003; 555: 126-133.

2. Richly H, Rape M, Braun S, Rumpf S, Hoege $\mathrm{C}$, Jentsch $\mathrm{S}$. A series of ubiquitin binding factors connects $\mathrm{CDC} 48 / \mathrm{p} 97$ to substrate multiubiquitylation and proteasomal targeting. Cell 2005; 120: 73-84.

3. Dalal S, Hanson PI. Membrane traffic: what drives the AAA motor? Cell 2001; 104: 5-8.

4. Zhang H, Wang Q, Kajino K, Greene MI. VCP, a weak ATPase involved in multiple cellular events, interacts physically with BRCA1 in the nucleus of living cells. DNA Cell Biol 2000; 19: 253-263.

5. Kondo H, Rabouille C, Newman R, Levine TP, Pappin D, Freemont $\mathrm{P}$ et al. p47 is a cofactor for p97-mediated membrane fusion. Nature 1997; 388: 75-78.

6. Meyer HH, Shorter JG, Seemann J, Pappin D, Warren G. A complex of mammalian ufd1 and npl4 links the AAA-ATPase, p97, to ubiquitin and nuclear transport pathways. EMBO J 2000; 19: 2181-2192.

7. Laco MN, Cortes L, Travis SM, Paulson HL, Rego AC. Valosin-containing protein (VCP/p97) is an activator of wild-type ataxin-3. PLoS One 2012; 7: e43563.

8. Yeung HO, Kloppsteck P, Niwa H, Isaacson RL, Matthews S, Zhang $X$ et al. Insights into adaptor binding to the AAA protein p97. Biochem Soc Trans 2008; 36(Pt 1): 62-67.

9. Wang Q, Song C, Li CC. Molecular perspectives on $\mathrm{p} 97-\mathrm{VCP}$ : progress in understanding its structure and diverse biological functions. J Struct Biol 2004; 146: 44-57.

10. Rabouille C, Levine TP, Peters JM, Warren G. An NSF-like ATPase, p97, and NSF mediate cisternal regrowth from mitotic Golgi fragments. Cell 1995; 82: 905-914.

11. Zhang SH, Liu J, Kobayashi R, Tonks NK. Identification of the cell cycle regulator VCP (p97/CDC48) as a substrate of the band 4.1-related protein-tyrosine phosphatase PTPH1. $J$ Biol Chem 1999; 274: 17806-17812.

12. Ye Y, Meyer HH, Rapoport TA. The AAA ATPase Cdc48/p97 and its partners transport proteins from the ER into the cytosol. Nature 2001; 414: 652-656.

13. Rabinovich E, Kerem A, Frohlich KU, Diamant N, Bar-Nun S. AAA-ATPase p97/Cdc48p, a cytosolic chaperone required for endoplasmic reticulum-associated protein degradation. Mol Cell Biol 2002; 22: 626-634.

14. Cao K, Nakajima R, Meyer HH, Zheng Y. The AAA-ATPase Cdc48/p97 regulates spindle disassembly at the end of mitosis. Cell 2003; 115: 355-367.

15. Acs K, Luijsterburg MS, Ackermann L, Salomons FA, Hoppe T, Dantuma NP. The AAA-ATPase VCP/p97 promotes 53BP1 recruitment by removing L3MBTL1 from DNA double-strand breaks. Nat Struct Mol Biol 2011; 18: 1345-1350.

16. Meerang M, Ritz D, Paliwal S, Garajova Z, Bosshard M, Mailand N et al. The ubiquitinselective segregase VCP/p97 orchestrates the response to DNA double-strand breaks. Nat Cell Biol 2011; 13: 1376-1382.

17. Livingstone $M$, Ruan $H$, Weiner J, Clauser KR, Strack $P$, Jin $S$ et al. Valosin-containing protein phosphorylation at Ser784 in response to DNA damage. Cancer Res 2005; 65: 7533-7540.

18. Chan DW, Mody CH, Ting NS, Lees-Miller SP. Purification and characterization of the double-stranded DNA-activated protein kinase, DNA-PK, from human placenta. Biochem Cell Biol 1996; 74: 67-73.

19. Lees-Miller SP. The DNA-dependent protein kinase, DNA-PK: 10 years and no ends in sight. Biochem Cell Biol 1996; 74: 503-512.

20. Kong X, Shen Y, Jiang N, Fei X, Mi J. Emerging roles of DNA-PK besides DNA repair. Cell Signal 23: 1273-1280.

21. Mi J, Dziegielewski J, Bolesta E, Brautigan DL, Larner JM. Activation of DNA-PK by ionizing radiation is mediated by protein phosphatase 6. PLOS ONE 2009; 4: e4395.

22. Wechsler T, Chen BP, Harper R, Morotomi-Yano K, Huang BC, Meek K et al. DNA-PKcs function regulated specifically by protein phosphatase 5. Proc Natl Acad Sci USA 2004; 101: $1247-1252$.

23. Douglas P, Moorhead GB, Ye R, Lees-Miller SP. Protein phosphatases regulate DNA-dependent protein kinase activity. J Biol Chem 2001; 276: 18992-18998.

24. Shen $Y$, Wang $Y$, Sheng $K$, Fei $X$, Guo $Q$, Larner $J$ et al. Serine/threonine protein phosphatase 6 modulates the radiation sensitivity of glioblastoma. Cell Death Dis 2011; 2: e241.

25. Griciuc A, Aron L, Piccoli $G$, Ueffing M. Clearance of rhodopsin(P23H) aggregates requires the ERAD effector VCP. Biochim Biophys Acta 2010; 1803: 424-434.

26. Wojcik C, Rowicka M, Kudlicki A, Nowis D, McConnell E, Kujawa M et al. Valosincontaining protein (p97) is a regulator of endoplasmic reticulum stress and of the degradation of $\mathrm{N}$-end rule and ubiquitin-fusion degradation pathway substrates in mammalian cells. Mol Biol Cell 2006; 17: 4606-4618.

27. Shao RG, Cao CX, Zhang H, Kohn KW, Wold MS, Pommier Y. Replication-mediated DNA damage by camptothecin induces phosphorylation of RPA by DNA-dependent protein kinase and dissociates RPA:DNA-PK complexes. EMBO J 1999; 18: 1397-1406.

28. Block WD, Yu Y, Lees-Miller SP. Phosphatidyl inositol 3-kinase-like serine/threonine protein kinases (PIKKs) are required for DNA damage-induced phosphorylation of the $32 \mathrm{kDa}$ subunit of replication protein A at threonine 21. Nucleic Acids Res 2004; 32: 997-1005.

29. Sakasai R, Shinohe K, Ichijima Y, Okita N, Shibata A, Asahina K et al. Differential involvement of phosphatidylinositol 3-kinase-related protein kinases in hyperphosphorylation 
of replication protein A2 in response to replication-mediated DNA double-strand breaks. Genes Cells 2006; 11: 237-246.

30. Hanzelmann $\mathrm{P}$, Schindelin $\mathrm{H}$. The structural and functional basis of the p97/VCPinteracting motif (VIM) mutually exclusive binding of cofactors to the N-terminal domain of p97. J Biol Chem 2011; 286: 38679-38690.

31. Tang WK, Li D, Li CC, Esser L, Dai R, Guo L et al. A novel ATP-dependent conformation in p97 N-D1 fragment revealed by crystal structures of disease-related mutants. EMBO J 2010; 29: 2217-2229.

32. Downward J. RNA interference libraries prove their worth in hunt for tumor suppressor genes. Cell 2005; 121: 813-815.

33. Kirchgessner CU, Patil CK, Evans JW, Cuomo CA, Fried LM, Carter T et al. DNAdependent kinase (p350) as a candidate gene for the murine SCID defect. Science 1995 267: 1178-1183.

34. Peterson SR, Kurimasa A, Oshimura M, Dynan WS, Bradbury EM, Chen DJ. Loss of the catalytic subunit of the DNA-dependent protein kinase in DNA double-strand-break-repair mutant mammalian cells. Proc Natl Acad Sci USA 1995; 92 3171-3174.

35. Moll U, Lau R, Sypes MA, Gupta MM, Anderson CW. DNA-PK, the DNA-activated protein kinase, is differentially expressed in normal and malignant human tissues. Oncogene 1999; 18: 3114-3126.

36. Douglas P, Zhong J, Ye R, Moorhead GB, Xu X, Lees-Miller SP. Protein phosphatase 6 interacts with the DNA-dependent protein kinase catalytic subunit and dephosphorylates gamma-H2AX. Mol Cell Biol 30: 1368-1381.

37. Wang $Q$, Gao F, Wang T, Flagg T, Deng X. A nonhomologous end-joining pathway is required for protein phosphatase $2 \mathrm{~A}$ promotion of DNA double-strand break repair Neoplasia 2009; 11: 1012-1021.
38. Wojcik C, Yano M, DeMartino GN. RNA interference of valosin-containing protein (VCP/p97) reveals multiple cellular roles linked to ubiquitin/proteasome-dependent proteolysis. J Cell Sci 2004; 117(Pt 2): 281-292.

39. Lu CC, Lin HF, Lee HS, Kao WY. Metastatic colon cancer presenting as Pancoast's disease. Am J Surg 2009; 197: e51-e52.

40. Ding Q, Reddy YV, Wang W, Woods T, Douglas P, Ramsden DA et al. Autophosphorylation of the catalytic subunit of the DNA-dependent protein kinase is required for efficient end processing during DNA double-strand break repair. Mol Cell Biol 2003; 23: 5836-5848.

41. Reddy YV, Ding Q, Lees-Miller SP, Meek K, Ramsden DA. Non-homologous end joining requires that the DNA-PK complex undergo an autophosphorylation-dependent rearrangement at DNA ends. J Biol Chem 2004; 279: 39408-39413.

42. Singh NP, McCoy MT, Tice RR, Schneider EL. A simple technique for quantitation of low levels of DNA damage in individual cells. Exp Cell Res 1988; 175: 184-191.

43. Klaude M, Eriksson S, Nygren J, Ahnstrom G. The comet assay: mechanisms and technical considerations. Mutat Res 1996; 363: 89-96.

44. Franken NA, Rodermond HM, Stap J, Haveman J, van Bree C. Clonogenic assay of cells in vitro. Nat Protoc 2006; 1: 2315-2319.

(c) (3) $\odot$ Cell Death and Disease is an open-access journal BY NC ND published by Nature Publishing Group. This work is licensed under a Creative Commons Attribution-NonCommercialNoDerivs 3.0 Unported License. To view a copy of this license, visit http://creativecommons.org/licenses/by-nc-nd/3.0/ 